\title{
Salud bucal en nativos Maká de 12 a 15 años, Mariano Roque Alonso, Paraguay.
}

\section{Resumen}

Objetivo: describir el estado de salud bucal en nativos Maká de 12 a 15 años de edad que residen en la ciudad de Mariano Roque Alonso, Paraguay. Materiales y métodos: Estudio observacional descriptivo de corte transversal en 59 niños de 12 a 15 años, evaluados por medio de la inspección oral y la aplicación de un cuestionario para indagar aspectos relativos a factores de riesgo presentes. Resultados: El Índice CPO-D fue de 10.5, se pudo constatar la ausencia del componente de piezas obturadas. El 94.9\% presentó gingivitis leve. Se halló una prevalencia de $30.5 \%$ de Fluorosis y lesiones no cariosas en un $11 \%$ de la población examinada. Conclusión: los resultados de los índices obtenidos para medir las condiciones bucales se encuentran por encima
Claudia Patricia Burgos Florentín ${ }^{1}$,

Jasmin Farjoud López ${ }^{1}$, Julia Mariel Florentín Servín ${ }^{1}$, Neida Yanina González Ayala ${ }^{1}$

Liz Tamara Martínez ${ }^{1}$,

María Belén Pérez Saucedo ${ }^{1}$,

Nohelia Pérez Bejarano²,

Domingo Ávalos ${ }^{2}$,

Marta Ferreira Gaona²,

Diana Sanabria ${ }^{2}$

de la meta establecida por la OMS; demuestrando un importante deterioro en el estado bucal de los niños nativos Maká.

Palabras clave: Salud bucal, nativos, caries dental, gingivitis, fluorosis dental, lesiones no cariosas, placa bacteriana.

\section{Artigo Original}

\section{Saúde Bucal em nativos Maká 12 a 15 anos, Mariano Roque Alonso, Paraguai.}

\section{Resumo}

Objetivo: Descrever a condição de saúde oral em nativos Maká entre 12 a 15 anos de idade residentes em Mariano Roque Alonso, Paraguai. Materiais e métodos: Estudo observacional descritivo de corte transversal em 59 crianças de 12 a 15 anos, avaliadas através do exame clínico e aplicação de um questionário para investigar questões relacionadas com fatores de risco. Resultados: $\mathrm{O}$ Índice CPO-D foi de 10.5, pôde-se constatar a ausência do componente de peças obturadas. O 94.9\% apresentou gengivite leve. Foi encontrada uma prevalência de 30.5\% de Fluorose e lesões não cariosas em $11 \%$ da população examinada. Conclusão: Os resultados obtidos, acima da meta estabelecida pela OMS; mostram uma deterioração significativa das condições de saúde bucal das crianças.

Palavras chave: Saúde bucal, população indígena, cárie dentária, gengivite, fluorose dentária, placa bacteriana. 


\section{Oral health in native Maká from 12 To 15 years old, Mariano Roque Alonso, Paraguay.}

\section{Abstract}

Aims: Describe the state of oral health in native Maka from 12 to 15 years old, Mariano Roque Alonso, Paraguay. Methods: Descriptive observational cross-sectional study in 59 children between 12 and 15 years old, evaluated through oral inspection and application of a questionnaire to investigate aspects of risk factors present. Results: The DMFT index was 10.5, this confirming the absence of sealed tooth, and $94.9 \%$ presented

\section{Introducción}

El adecuado estado de la salud bucal permite mantener funciones básicas para cada ser humano, como la alimentación y la comunicación. Sin embargo, desde las primeras etapas del desarrollo, pueden presentarse factores que afectan su buen funcionamiento, preservación e integridad. Las patologías bucales tales como caries, gingivitis, fluorosis y lesiones no cariosas afectan en mayor o menor medida a las poblaciones, siendo los infantes los mas afectados por las primera, y de ahí la importancia de identificar la presencia de factores de riesgo asociados a ellas ${ }^{1}$.

Las condiciones de salud bucal a nivel individual y colectivo son evaluadas principalmente con análisis de ocurrencia de caries y dolencias periodontales. Desde el punto de vista epidemiológico, la caries ha sido más estudiada con el objeto de cono- mild gingivitis. A prevalence of $30.5 \%$ of fluorosis and non-carious lesions in $11 \%$ of the population examined was found. Conclusion: The results showed a significant deterioration in the oral status of the Maka children due to the increased prevalence and higher caries index compared toa the goal set by WHO.

Keywords: Oral Health, native, dental caries, gingivitis, dental fluorosis, non-carious lesions, plaque.

cer mejor sus determinantes, prevalencia y distribución en las poblaciones; de manera a establecer las medidas preventivas adecuadas2. De acuerdo a la Organización Mundial de la Salud y la Federación Dental Internacional, la caries y la gingivitis son las patologías bucales de mayor prevalencia, magnitud o gravedad de acuerdo a condiciones socioeconómicas. En países desarrollados esta prevalencia oscila entre 60 y $90 \%$ y en países en vías de desarrollo entre 90 y $98 \% 3$.

\section{Caries y gingivitis}

La caries es conocida como una enfermedad infecciosa, universal, multifactorial y crónica; su avance natural afecta la estructura del diente, provocando una lesión irreversible en su estado avanzado. Su 
presencia se debe a un desequilibrio entre agentes y la intensidad de la respuesta biológica del hospedador y se inicia en boca mucho tiempo antes de manifestarse en forma de lesiones visibles 4-7. Por su parte, la gingivitis, es considerada la entidad más común de las enfermedades periodontales, con tasa de prevalencia muy alta y segunda causa de morbilidad bucal y comparte con la caries la misma entidad idiopática: la placa microbiana, dónde difieren en la calidad de los microorganismos especializados en el ataque a diferentes tipos de tejidos ${ }^{6,8}$.

Por lo expuesto, para el desarrollo de ambas patologías es necesaria la presencia de placa microbiana en niveles superiores a los compatibles con salud (hasta 20\% evaluado a través del Índice de O'Leary), es así que para la caries, los carbohidratos de la dieta sirven de base para la producción de ácidos orgánicos y como fuente para el desarrollo de la matriz de la placa; por ello la frecuencia de su ingestión y el tipo, son componentes importante, para el desarrollo de caries, sin tener este factor mucha importancia para el desarrollo de enfermedades periodontales ${ }^{14}$.

Es entonces un hecho que la deficiente higiene bucal facilita la organización de la placa, jugando un papel fundamental para el inicio y progresión de ambas patologías. Este hecho se encuentra influenciado por el conocimiento sobre cuidados de la salud oral y la condición socioeconómica, que determinan el estilo de vida, autocuidado de la salud de las personas y presencia de factores biológicos (anomalías dentarias) $^{6,8,9}$.

\section{Índices}

Existen índices utilizados para evaluar varias de las patologías bucales. La presencia y gravedad de la caries en un individuo o población, popularmente se utiliza el Índice CPO-D (desarrollado por Klein, Palmer y Knutson en 1935), entre otros índices. La enfermedad gingival, pueden ser evaluadas también a través del Índice Gingival de Sillness y Löe, en el cual se puede optar por evaluar las piezas de referencia según Ramfjord ${ }^{10}$.

Otras patologías como la fluorosis dental y lesiones no cariosas producen una serie de cambios clínicos y defectos estructurales considerados como condiciones irreversibles. La primera, causada por la ingestión crónica de niveles elevados de fluoruros durante la formación del diente, cuya severidad depende del tiempo de erupción de las piezas dentarias, cantidad y concentración de fluoruro ingerido y duración de la exposición15,16. Las segundas (la abrasión, erosión, abfracción y la atrición), de etiología no bacteriana, pueden ser causadas por hábitos, fuerzas para funcionales o desórdenes en el sistema gástrico, que causan regurgitación, produciendo una pérdida de sustancia mineral de superficie dentaria generando síntomas de hipersensibilidad en etapas avanzadas ${ }^{17,18}$.

Para medir la afectación debida a exposición al flúor, se puede usar el Índice de Dean, que establece cinco grados, según la extensión de la lesión (normal, cuestionable, muy leve, leve, moderado y severo) ${ }^{10,11,12}$. Como otras poblaciones, las poblaciones indígenas pueden verse afectadas por estas patologías, sus creencias y prácticas referentes a la salud determinan la frecuencia de aparición de los factores de riesgo asociados en cada grupo étnico; así como los recursos comunitarios para la promoción, prevención o cura de enfermedades bucales ${ }^{2,3,19,20}$. 


\section{Descripción demográfica}

Según la Dirección General de Estadísticas Encuestas y Censo (DGEEC) la población indígena en Paraguay asciende a 108.308 personas en el año 2008.Entre otras etnias los Maká son originarios del Chaco Paraguayo y pertenecen a la familia lingüística Mataco Mataguayo. Se divididen en tres comunidades, distribuidas en Itapúa, Alto Paraná y Central; esta última con 890 habitantes. La lengua Maká es la más hablada entre ellos $(96,4 \%)$ y actualmente residen en la localidad de Mariano Roque Alonso (Dpto. Central), desde el año 1985 en un predio de una zona urbanizada ${ }^{23,24}$.

Este grupo de indígenas originalmente, se dedicaban a la caza, la pesca y la recolección, pero cambiaron su estilo de vida para adaptarse al nuevo entorno posterior a su migración interna y en la actualidad se dedican casi exclusivamente a la artesanía, que constituye su principal medio de subsistencia ${ }^{25,26}$.

Este estudio se realizó con el propósito de describir el estado de salud bucal en nativos Maká de 12 a 15 años de edad que residen en la ciudad de Mariano Roque Alonso (Paraguay), por medio de la inspección oral y la aplicación de un cuestionario para indagar aspectos relativos a los factores de riesgo presentes.

\section{Material y métodos}

Se realizó un estudio observacional descriptivo de corte transversal en 59 niños de 12 a 15 años que asistían en la Escuela № 2490 Ángel Kanaití (Comunidad Maká), en la localidad de Mariano Roque Alonso.
Para la selección de la muestra se realizó un muestreo no probabilístico de casos consecutivos. El cálculo del tamaño de la muestra se efectuó con un 95\% de confianza en base a una frecuencia probable de 50\% en una población de 69 individuos y un ajuste del $10 \%$ para las probables no respuestas. Para el acceso a la población, se solicitó autorización a las personas responsables, incluyendo el Cacique de la comunidad, supervisión educativa de la zona, docentes de los distintos grados y finalmente a los padres de los niños.

A éstos últimos, y complementando los procedimientos éticos, se les distribuyó la hoja de información (en español y Maká) y el consentimiento informado. El trabajo fue aprobado por el Comité de Ética en la investigación de la Universidad del Pacífico Privada.

Para la recolección de datos se diseñó una ficha, que constaba de secciones para el registro de datos sociodemográficos, factores de riesgo y odontograma. Para el examen clínico se utilizó luz artificial combinada con luz natural.

La inspección intraoral se realizó con espejo bucal plano $\mathrm{n}^{\circ} 5$, aislamiento relativo, sonda periodontal calibrada de la OMS, tipo IPC (Hu Frie$\mathrm{dy}^{\mathrm{TM}}$ o Trinity ${ }^{\circledR}$ ) y revelador de placa (Test Plac (R).Una vez terminada la inspección, se procedió a la entrega a cada niño de un kit compuesto por cepillo yw pasta dental que sirvieron para poner en práctica la instrucción en técnica de cepillado que se dio después de la inspección. Los datos fueron cargados en una base datos de EXCEL 97 - 2003 de la Microsoft y analizados a través del programa Epi Info ${ }^{\mathrm{TM}}$ versión 3.5.1, desarrollado por el Centers For Disease Control And Prevention (CDC). 


\begin{tabular}{|c|c|c|c|}
\hline \multirow{2}{*}{ Edad } & \multicolumn{2}{|c|}{ CPO-D } & \multirow{2}{*}{$\begin{array}{c}\text { Población } \\
\text { total }\end{array}$} \\
\cline { 2 - 3 } & Masculino & Femenino & \\
\hline 12 & 8.2 & 7.0 & 7.6 \\
\hline 13 & 9.4 & 15.0 & 12.4 \\
\hline 14 & 9.2 & 11.8 & 10.6 \\
\hline 15 & 9.7 & 13.3 & 11.5 \\
\hline Total & 9.1 & 11.8 & 10.5 \\
\hline
\end{tabular}

Tabla 1. Índice CPO-D según edad y sexo.Comunidad Maká. Mariano Roque Alonso.

\section{Resultados}

\section{Caries}

Fueron evaluados 59 niños entre 12 y 15 años, cuya mediana de edad fue de 13 años, el 51 \% correspondía al sexo femenino.En cuanto a las características clínicas de la población, el CPO-D fue de 10.5 para la población total con valores más elevados en las mujeres (CPO-D 11.8) y la edad más afectada fue la de 15 años (CPO-D 11.5). Se puede observar que existe un incremento en los valores del índice, conforme aumenta la edad. (Tabla 1)

\begin{tabular}{|l|c|c|c|c|c|c|}
\hline \multirow{2}{*}{$\begin{array}{l}\text { Niveles de } \\
\text { gravedad }\end{array}$} & \multicolumn{2}{|c|}{ Masculino } & \multicolumn{2}{|c|}{ Femenino } & \multicolumn{2}{|c|}{ Total } \\
\cline { 2 - 7 } & No & $\%$ & No & $\%$ & No & $\%$ \\
\hline Leve & 17 & 58.6 & 20 & 66.7 & 37 & 62.7 \\
\hline Moderado & 10 & 34.5 & 8 & 26.7 & 18 & 30.5 \\
\hline Normal & 2 & 6.9 & 1 & 3.3 & 3 & 5.1 \\
\hline Severo & - & - & 1 & 3.3 & 1 & 1.7 \\
\hline Total & 29 & 100.0 & 30 & 100.0 & 59 & 100.0 \\
\hline
\end{tabular}

Tabla 2. Niveles de severidad de la inflamación gingival (Índice Gingival de Sillnes y Löe) y según sexo. Comunidad Maká, Mariano Roque Alonso.

\begin{tabular}{|l|c|c|}
\hline Grados de severidad & No & $\%$ \\
\hline Muy Leve & 9 & 15.30 \\
\hline Cuestionable & 1 & 1,70 \\
\hline Leve & 7 & 11.90 \\
\hline Moderado & 1 & 1.70 \\
\hline No presenta & 41 & 69.50 \\
\hline Total & 59 & 100.0 \\
\hline
\end{tabular}

Tabla 3. Grados de severidad de Fluorosis (Índice de Dean). Comunidad Maká, Mariano Roque Alonso.

\section{Gingivitis}

El índice gingival de Sillness y Löe señaló que, respecto a niveles de gravedad de la inflación gingival, predominó el grado leve $(62.7 \%)$ de la población total, donde el grado severo fue visto mayormente en el sexo femenino $(3.3 \%)$, siendo la distribución por sexo equitativa para ambos (Tabla 2 ).

\section{Fluorosis}

La prevalencia de fluorosis en la población fue baja, presentándose sólo en un 30,5\% de la misma, el grado de severidad predominante fue muy leve $15.3 \%(9)$ teniendo en cuenta la escala Dean (0: normal, 1: cuestionable, 2: muy leve, 3:leve, 4:moderado, 5:severo) ${ }^{12}$ (Tabla 3).

\begin{tabular}{|l|c|c|}
\hline Lesiones no cariosas & No & $\%$ \\
\hline No & 49 & 83.1 \\
\hline $\mathrm{Si}$ & 10 & 16.90 \\
\hline Total & 59 & 100.0 \\
\hline
\end{tabular}

Tabla 4. Presencia de lesiones no cariosas. Comunidad Maká, Mariano Roque Alonso. 


\section{Lesiones no cariosas}

Se observó que la presencia de lesiones no cariosas afectó a solo el 17\% de la muestra, y la existencia de hábitos deletéreos tales como la onicofagia; se presentó en el 54\% (Tabla 4).

\section{Factores de riesgo}

En cuanto a los factores de riesgo asociados a las patologías evaluadas, la deficiencia en cuanto a higiene bucal fue demostrada por el Índice de O'Leary con un valor de $83 \%$ como promedio; ubicando a la población dentro del grupo de alto riesgo para caries y enfermedad periodontal (Nivel bajo: $10-20 \%$ nivel medio: $21-50 \%$ nivel alto: $51 \%$ y más).Resultado de la encuesta, sólo el $66 \%$ de la población refirió realizar la práctica del cepillado dental, de éstos un 39\% lo hace al menos una vez al día con cepillo y pasta dental. El $34 \%$ de los evaluados (refirió no haber recibido instrucción acerca de las técnicas de higiene bucal. El 61\% (36) de los evaluados respondió que no asisten a consulta dental (Tabla 5).

\section{Discusión}

La frecuencia de caries en las poblaciones indígenas reportada por estudios en Latinoamérica, fue variando a lo largo del tiempo, probablemente debido a la incorporación de alimentos industrializados como consecuencia de la escasez de los recursos naturales en su hábitat original, generando la migración de algunas tribus a zonas urbanizadas. No se encontraron publicaciones que indiquen la situación bucal de las poblaciones indígenas en el Paraguay a diferencia de estudios realizados en Brasil y en otros países que las documentan en sus respectivas poblaciones. El hallazgo del Índice CPO-D que se obtuvo en los niños Maká, fue muy

\begin{tabular}{|l|l|l|}
\hline \multicolumn{1}{|c|}{ Factores de Riesgo } & $\mathbf{N}^{\circ}$ & \multicolumn{1}{l|}{$\%$} \\
\hline Higiene bucal & 39 & 66.1 \\
\hline siempre & 13 & 22.0 \\
\hline nunca & 7 & 11.9 \\
\hline a veces & \multicolumn{2}{|l|}{} \\
\hline Consumo de carbohidratos sólidos \\
\hline De vez en cua & 31 & 52.5 \\
\hline Todos los dìas & 19 & 32.2 \\
\hline No consume & 9 & 15.3 \\
\hline Consumo de bebidas azucaradas & \multicolumn{2}{|l}{} \\
\hline Gaseosas & 39 & 66.1 \\
\hline Jugos artificiales & 5 & 8.5 \\
\hline Jugos naturales & 11 & 18.6 \\
\hline Todas & 1 & 1.7 \\
\hline Gaseosas jugos artificiales & 2 & 3.4 \\
\hline No responde & 1 & 1.7 \\
\hline $\begin{array}{l}\text { Conocimiento de atención } \\
\text { odontológica }\end{array}$ & \multicolumn{2}{|l}{} \\
\hline si & 17 & 28.8 \\
\hline no & 42 & 71.2 \\
\hline Acude al odontólogo & 39.0 \\
\hline si & 61.0 \\
\hline no & \multicolumn{2}{|l}{} \\
\hline
\end{tabular}

Tabla 3. Grados de severidad de Fluorosis (Índice de Dean). Comunidad Maká, Mariano Roque Alonso.

alto $(10,2)$ de acuerdo a los niveles de severidad establecidos por la OMS (3,0 para los 12 años) y resultó mayor a los encontrados por Arantes et al. ${ }^{2}$ (CPO-D 3,7), así como Linares et $\mathrm{al}^{29}$ (CPO-D 3,18), y Sampaio FC et al. (CPO-D 3,18) ${ }^{30}$. Todos las anteriores investigaciones fueron realizadas en nativos infantes de diferentes etnias de Brasil en edades que oscilaban entre los 12 y 15 años.

En comparación con la población no indígena en Paraguay; el Índice CPO-D 6,2 hallado por Ferreira $\mathrm{M}^{31}$, corresponde a niños de la misma edad y sin hogar, habitando en situaciones de extrema po- 
breza en las ciudades del departamento Central. Este estudio muestra un índice elevado pero inferior al hallado en la comunidad Maká, al igual que en el estudio de Yankilevich32 en poblaciones no indígenas de la región metropolitana donde el índice también fue inferior. Por su parte en estudios hechos en Colombia como el de Triana et al. ${ }^{+}$, el año 2005, se halló un CPO D de 1,34 para la edad de 12 años, muy inferior al hallado en este estudio.

La prevalencia de fluorosis fue de $30,5 \%$ en los niños Maká, siendo elevado en comparación a estudios en niños no nativos, como el realizado por Ferreira M31, en el cual fue de 11,1 \%, Triana et al.19 en Colombia, en niños escolares indígenas, donde se demostró la ausencia de esta patología y en Brasil Carvalho et al.33 en una población no indígena, donde se encontró una prevalencia del 29, $2 \%$, cifra similar al hallazgo de este estudio.

En cuanto al estado gingival, el $94,6 \%$ de la población presentó cierto grado de inflamación, contrastando con el estudio de Ferreira M31 el cual encontró gingivitis solo en el 17, 8\%. De igual manera, Triana et al ${ }^{19}$ encontraron a 40,3\% de niños con salud gingival afectada. Todos estos datos provistos por otros estudios en países latinoamericanos que en nuestro país poco se conoce acerca del estado de salud bucal en nativos, y en contraste, en países como Brasil se puede observar que el conocimiento en este tema ha apoyado una disminución en los niveles de caries en muchas comunidades amazónicas en las últimas tres décadas2, pudiendo deberse al aumento en la exposición del esmalte al fluoruro en sus diversas formas ${ }^{19}$.

En contraparte, la documentación del deterioro de las condiciones de salud bucal y una tendencia al aumento de prevalencia de caries suponen los autores que pudiera ser debido al aumento del consumo de alimentos industrializados, (azúcar y otros tipos de carbohidratos fermentativos), considerando que los pueblos indígenas en su entorno natural, tendrían dietas tradicionales, con alto contenido en fibra y bajo en hidratos de carbono 2,21 así pues, la susceptibilidad a la caries aumentaría 35 veces.

La combinación de un aumento del consumo de alimentos azucarados y la disminución de los alimentos tradicionales trae consigo el aumento de caries y enfermedad periodontal, que se ve agravado a su vez por otros factores como la falta de asistencia sanitaria y el alto costo que implica un tratamiento dental, además de las modificaciones socioeconómicas y ambientales sumada a la falta de programas preventivos $^{21,22}$.

\section{Conclusión}

Los resultados obtenidos, demuestran un importante deterioro en el estado bucal de los niños nativos de la comunidad Maká, demostrado con alto nivel de caries (por encima de la meta establecida por la OMS), presencia de placa y una dieta rica en azúcares. Se recomienda dirigir las acciones preventivas a estos pueblos originarios que migran al cinturón urbano, haciendo campañas enfocadas a la promoción en salud destacando los posibles daños que trae consigo la mala praxis en hábitos de higiene y dieta, de manera que la adaptación a nuevas costumbres en entornos urbanos les acercarán a mejorar su estado de salud bucal actual, además de motivar el acercamiento a la asistencia odontológica para tratamientos con énfasis en la limitación del daño y rehabilitación. 


\section{Referencias bibliográficas}

1. Ortega-Maldonado M, Mota-Sanhua V, López-Vivanco JC. Oral health status of adolescents in México City. Revista de Salud Pública. Sep 2007;9(3):380-7.

2. Arantes R, Santos RV, Coimbra Jr CE. Oral health among the Xavánte Indians in Pimentel Barbosa, Mato Grosso, Brazil. Cad saúde pública. Mar 2001;17(2):375-84.

3. Iglesias-Padrón CV, Arenas-Monreal L, Bonilla-Fernández P, Cruz-Gama E. Intervención educativa en salud bucal con preescolares indígenas de México. Revista ADM 2008;LXV(5):247-252.

4. Barrancos Mooney J, Barrancos PJ. Operatoria dental: integración clínica. 4a ed. Buenos Aires: Médica Panamericana; 2006.1345 p. 5. Negroni M. Microbiología estomatológica: fundamentos y guía práctica. Buenos. Aires: Médica Panamericana; 1999.566 p. 6. Ferro Camargo MB, Gómez Guzmán M. Fundamentos de la Odontología: periodoncia. 2a ed. Pontificia Universidad Javeriana: Bogotá; 2007.

7. De la Cruz Rubianes E, Evangelista Rodriguez J, Gil Mori L, Ignacion Leon E, Milla Torres D, Monge Poma V, et al. Determinacion de riesgo estomatologico de niños de 5-12 años que acuden a la Clínica de Pregrado de la Facultad de Odontología de UNMSM en el año 2010 [Internet]. Salcedo R, editor. Lima-Perú: Universidad Nacional Mayor de San Marcos; 2008 [citado 25 sept 2012]. 27 p. Recuperado a partir de: https://es.scribd.com/doc/130776735/determinacion-deriesgo-estomatologico-de-ninos-de-5-12-anos

8. Murrieta-Pruneda JF, Juárez-López LA, Linares-Vieyra C, Zurita-Murillo V, Meléndez-Ocampo AF, Ávila-Martínez CR, et al. Prevalencia de gingivitis asociada a la higiene oral, ingreso familiar y tiempo transcurrido desde la última consulta dental, en un grupo de adolescentes de Iztapalapa, Ciudad de México. Boletín médico del Hospital Infantil de México. octubre de 2008;65(5):367-75.

9. Mursulí M, Rodríguez, Landa L, Hernández M. Anomalías dentarias. Gaceta Médica Espirituana[Internet] 2006 [citado 29 Jun 2011];8(1).Disponible en: http://bvs.sld.cu/revistas/gme/pub/vol.8.(1)_12/p12.html

10. Lindhe J. Periodontología Clínica e Implantología Odontológica. Editorial Panamericana. 5o ed. Tomo I España; 2009

11. Fernández Pratts MJ, González-Longoria M de la CB, Castro Bernal C, Vallard Jímenez E, Lezama Flores G, Carrasco Gutiérrez R. Índices epidemiológicos para medir la caries dental. Red de Estomatología Social [Internet]. 13-jun-2011 [citado 7 de septiembre de 2015]. Disponible en: http://estsocial.sld.cu/docs/Publicaciones/Indices\%20epidemiologicos\%20para\%20 medir\%20la\%20caries\%20dental.pdf

12. Rioboo R. Higiene y prevención en odontología: Individual y comunitaria. Madrid: Avances Médico-Dentales; 1994.

13. Herazo B. Clínica del sano en odontología. Colombia: Ecoe; 2003.

14. Gómez D. Normas oficiales venezolanas del programa Nacional de salud oral. 2000 [citado 6 julio 2011] Disponible en: http://www.sld.cu/galerias/pdf/uvs/saludbucal/normasvenez.pdf

15. Gato H, Fuentes I, Duque de Estrada R, Hernández F, Zamora J. Fluorosis dental: no solo un problema estético. Rev Cubana Estomatol [Internet]. Dic 2007 [citado 06 Jul 2010]; 44(4). Disponible en: http://scielo.sld.cu/scielo.php?script=sci_ arttext\&pid=S0034-75072007000400014\&lng=es.)

16. Valadez M, Rodríguez F, Sánchez M. Prevalencia de Fluorosis dental en escolares de 6 a 15 años de edad. Revista Médica del IMSS. Ene 2008;46(1):67-72.

17. Cuniberti N, Rossi G. Lesiones cervicales no cariosas. La lesión dental del futuro. Actualizaciones Odontológicas. 2009;1-7.

18. Cendoya P, Hernández J, Dufeu E. Análisis computacional de lesiones cervicales no cariosas en un premolar superior Ingeniare Revista chilena de ingeniería. agosto de 2007;15(2):169-73.

19. Triana FE, Rivera SV, Soto L, Bedoya A. Estudio de morbilidad oral en niños escolares de una población de indígenas amazónicos. Colombia Médica. 2005;36(4 Supl 3):26-30.

20. Organización Panamericana de la Salud. Salud de los pueblos indígenas de América: evaluación de los logros en salud en el marco del Decenio Internacional de los pueblos indígenas del mundo. Washington. Organización Mundial de la Salud. 2008.332 p.21- Brandao Hirooka L. Condições de saúde bucal em pares mãe-filho na população indígena do Medio e Bajo Xingú: cárie dentária e necessidade de tratamiento. Riberão Preto (SP) [tesis de maestría]. [Riberão Preto]: Universidad de São Paulo; 2010.100 p. 21. Brandao Hirooka L. Condições de saúde bucal em pares mãe. filho na população indígena do Medio e Bajo Xingú: cárie dentária e necessidade de tratamiento. Riberão Preto (SP) [tesis de maestría]. [Riberão Preto]: Universidad de São Paulo; 2010. 100 p. indígenas. Londres. Survival International. 2008. 59 p. 
Burgos Florentín CP, Farjoud López J, Florentín Servín JM, González Ayala NY, Martínez LT,

Pérez Saucedo MB, Pérez Bejarano N, Ávalos D, Ferreira Gaona M, Sanabria D.

22. Woodman J, Grig S. El progreso puede matar. Como el desarrollo impuesto destruye la salud de los pueblos indígenas. Londres. Survival International. 2008. 59 p.

23. Dirección General Estadísticas, Encuestas y Censos. II Censo Nacional Indígena de Población y Viviendas. Asunción. Presidencia de la República; 2002. 30 p.

24. Zanardini J, Biedermann W. Los indígenas del Paraguay. Paraguay. Palo Santo; 2001. 252 p.

25. Comunidad Indígena Maká, una población que lucha por evitar su extinción. Roque Alonso Digital [14 de noviembre 2010] Disponible en: http://roquealonsodigital.com/?page_id=62

26. Schmidt PM, Peterson MJ. Biodiversity conservation and indigenous land management in the era of self. determination. Conserv Biol. diciembre de 2009;23(6):1458. 66.H

27. Abad González L. Salud intercultural y pueblos indígenas: la experiencia de un programa de salud de atención primaria con Comunidades Aguarunas de la selva amazónica en Perú. En: Fernández Juárez G, editor. Salud e interculturalidad en América Latina: perspectivas antropológicas. Quito. Ecuador: Abya. Yala; 2004. p 75. 92.

28. Hulley S, Cummings S, Browner S, Grady D, Newman T. Diseño de las investigaciones clínicas. 3ra ed. Barcelona: Lippincott; 2008.422 p.

29. Linares Lizarazo L, Lizarazo LRL, Paredes NRR, Herrera D, Gómez RÁ. Caries dental y condiciones de higiene oral en niños indigenas sikuani, municipio de Puerto Gaitan. Rev. Colomb. Investig. Odont. [Internet]. 15 de septiembre de 2010; 1(2). Recuperado a partir de: http://www.rcio.org/index.php/rcio/article/view/7

30. Chorreia Sampaio F, Soares de Morais Freitas CH, Barbosa de Farias Cabral M, de Azevedo Britto Machado AT. Dental caries and treatment needs among indigenous people of the Potiguara Indian reservation in Brazil.

Rev Panam Salud Publica. abril de 2010;27(4):246. 51.

31. Ferreira Gaona MI. Evaluación del estado de salud buco dental en menores en situación de calle, asistidos por la Fundación de Ayuda Republicana (FUNDAR) Paraguay: 2009. 2010. Mem. Inst. Investig. Cienc. Salud. Junio 2011;9(1): 21. 34.

32. Yankilevich ERLM de, Battellino LJ. Prevalence of dental caries in schoolchildren in a metropolitan region of the Córdoba Province, Argentina. Revista de Saúde Pública. diciembre de 1992;26(6):405. 13.

33. Carvalho TS, Kehrle HM, Sampaio FC. Prevalence and severity of dental fluorosis among students from João Pessoa, PB, Brazil. Brazilian Oral Research. septiembre de 2007;21(3):198. 203.

Recibido: $21 / 01 / 16$

Aceptado: 28/02/16

Correspondencia: Nohelia María Pérez Bejarano: dra.nohe@gmail.com

$(+595985500134)$ 